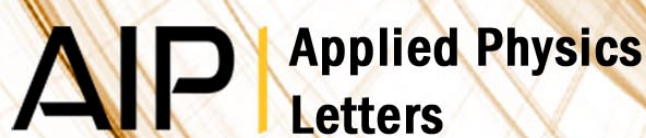

\section{Broadband and low loss high refractive index metamaterials in the microwave regime}

T. Campbell, A. P. Hibbins, J. R. Sambles, and I. R. Hooper

Citation: Appl. Phys. Lett. 102, 091108 (2013); doi: 10.1063/1.4794088

View online: http://dx.doi.org/10.1063/1.4794088

View Table of Contents: http://apl.aip.org/resource/1/APPLAB/v102/i9

Published by the American Institute of Physics.

\section{Additional information on Appl. Phys. Lett.}

Journal Homepage: http://apl.aip.org/

Journal Information: http://apl.aip.org/about/about_the_journal

Top downloads: http://apl.aip.org/features/most_downloaded

Information for Authors: http://apl.aip.org/authors

\section{ADVERTISEMENT}

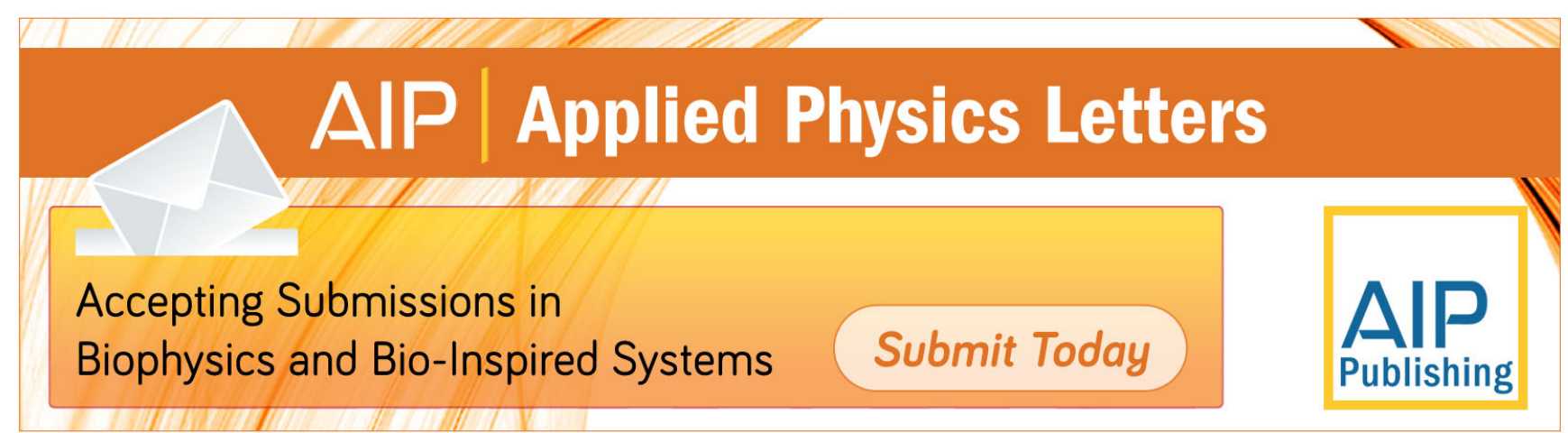




\title{
Broadband and low loss high refractive index metamaterials in the microwave regime
}

\author{
T. Campbell, ${ }^{1}$ A. P. Hibbins, ${ }^{2}$ J. R. Sambles, ${ }^{2}$ and I. R. Hooper ${ }^{2}$ \\ ${ }^{1}$ BAE systems, Military Air and Information, Warton Aerodrome, Warton, Lancashire PR4 IAX, \\ United Kingdom \\ ${ }^{2}$ Electromagnetic and Acoustic Materials Group, Department of Physics and Astronomy, University of Exeter, \\ Exeter EX4 4QL, United Kingdom
}

(Received 14 November 2012; accepted 19 February 2013; published online 6 March 2013)

\begin{abstract}
A broadband, low-loss, isotropic, high refractive index metamaterial comprising an array of close-packed cubic elements of cross-linked square metal plates is demonstrated in the microwave regime. The structuring of the cubic elements reduces the diamagnetic response exhibited by arrays of solid metal cubes, whilst maintaining the strongly enhanced permittivity arising from capacitative coupling between adjacent metal elements. The corresponding increase in refractive index can be tailored through appropriate structuring of the faces and/or spacing of the cubes. (C) 2013 American Institute of Physics. [http://dx.doi.org/10.1063/1.4794088]
\end{abstract}

Metamaterials are synthetic engineered electromagnetic composites with tailored values of permittivity and permeability. ${ }^{1-3}$ Whereas naturally occurring materials gain their electromagnetic properties from the interaction of the electric and magnetic fields with their individual atoms or molecules, a metamaterial gains its properties from artificially created subwavelength elements. The design of these "artificial atoms" permits effective permittivity and permeability values not readily found in nature, including such unusual properties as negative refractive indices. ${ }^{4-7}$ However, in order to achieve these properties, the individual elements of the metamaterial are frequently designed to be resonant and are, therefore, inherently narrowband and lossy. The more pragmatic goal of developing materials with structurally designed high positive refractive indices has, in our view, been under-studied, especially when one considers that some optical devices designed under the banner of transformation optics ${ }^{8-10}$ can be designed using precisely such material properties.

Previous work on high refractive index metamaterials has shown that a sub-wavelength array of parallel plate capacitors exhibits a non-resonant (and therefore broadband) enhancement of the effective permittivity, ${ }^{1,12}$ but is simultaneously strongly diamagnetic $(0<\mu \ll 1)$. Consequently, the refractive index of such systems $(n=\sqrt{\varepsilon} \sqrt{\mu})$ is not greatly enhanced. The three-dimensional equivalent to the array of parallel plate capacitors is a close-packed array of metallic cubes, and Wood and Pendry ${ }^{13}$ demonstrated that the diamagnetic response of such an array is a result of induced current loops that traverse the cubes and oppose the applied field.

In 2009, Shin et al. ${ }^{14}$ demonstrated numerically that, by appropriate structuring of the cubic elements, one might limit the area enclosed by the induced surface currents and reduce the diamagnetic response. Since the capacitative coupling between the elements can be maintained, a significant increase of the effective refractive index of the structure can be achieved. Their proposed structure consisted of an array of cubic elements of cross-linked square metal plates. Though the mechanisms proposed have been used to generate a two-dimensional material in the terahertz regime, ${ }^{15}$ there has been no demonstration of a three-dimensional structure.
In this work, an experimental demonstration of a broadband, isotropic, high refractive index metamaterial is presented. To characterise the electromagnetic response of the cubic samples experimentally, a stripline method for measuring the permittivity and permeability of metamaterials was developed. Stripline methods for material characterisation were pioneered by Barry in $1986^{16}$ in a paper in which he described the arrangement of a flat central conducting line symmetrically bounded by ground planes in a geometry such that the impedance is maintained at $50 \Omega$ across the frequency range of interest. The guided wave supported between the signal line and ground planes is transverse electric and magnetic (TEM) in nature and is polarised with the electric field orthogonal to the central conductor surface. Since there is no low frequency cut-off for such a guided wave, it is supported over a broad frequency range. When the stripline is connected to a calibrated vector network analyser (VNA) and a sample of unknown permittivity and permeability is placed symmetrically between the central conductor and ground planes, the complex reflection and transmission amplitude coefficients can be measured. The system can be considered equivalent to the case of electromagnetic radiation normally incident upon a thin slab, where the $50 \Omega$ lines are "free space" and the material under test is the thin slab of material, and can be described using the well-known thin-layer Fresnel equation. Given the complex reflection and transmission amplitude coefficients, the frequency, and the thickness of the sample, simple algebraic manipulation of Fresnel's equations allows the complex permittivity and permeability of the sample to be determined (this extraction method is commonly known as the Nicholson, Ross, Weir (NRW) method ${ }^{17,18}$ ).

Though this basic stripline method is, in general, simple and applicable to a broad range of sample types, there are some specific problems when using it to study metamaterials. First, the thickness of the metamaterial sample must correspond to the gap between the central conductor and the ground planes. Although for most naturally occurring materials, it is trivial to prepare a suitable sample to fit the gap, for metamaterial samples this may not be possible due to the 
size of the individual elements. Therefore, the gap size must be an integer multiple of the unit cell dimension. This condition, however, has an added benefit: the boundary condition imposed by the metallic boundaries forces the fields on either side of the sample (in the polarisation direction) to be identical-exactly the same condition as is imposed at the boundaries of a unit cell in a periodic sample upon normally incident plane wave illumination. This means that a sample only a single unit cell in height placed within such a stripline will result in the same reflection and transmission properties as for plane wave illumination of a bulk sample.

The second issue with the use of a stripline to measure metamaterial properties arises due to instabilities of the NRW extraction method when the relative impedance $\left(Z=\sqrt{\mu_{r} / \varepsilon_{r}}\right)$ of the sample under test is either close to unity or very large. When this is the case, either the reflection or the transmission coefficient is close to zero and experimental uncertainties in the measurements begin to dominate the extracted permittivities and permeabilities. The solid metal cubic structure described above suffers from exactly this problem since the permittivity is expected to be very large $(\varepsilon \gg 1)$ with a simultaneously very small permeability $(\mu \ll 1)$, leading to a large impedance mismatch and a correspondingly small transmission coefficient. In order to show how these limitations of stripline measurements can be overcome, we begin by considering the microwave response of an array of solid metal cubes, before demonstrating that appropriate structuring of such cubes can indeed result in much higher refractive indices.

Sixty copper cubes of side length $4.2 \mathrm{~mm}$ were fabricated and $85 \mu \mathrm{m}$ thick acrylic stickers $(\varepsilon=2.55+0.001 \mathrm{i}$ at $10 \mathrm{GHz}$ ) attached on each face to ensure uniform separation (inset Figure 1). These cubes were arranged into two close packed arrays of $1 \times 5 \times 6$ cubes and bound together with a thin band of polyolefin heat shrink (numerical modeling ${ }^{19}$ showed that the thin band of polyolefin heat shrink had very little effect on the electromagnetic response of the array).

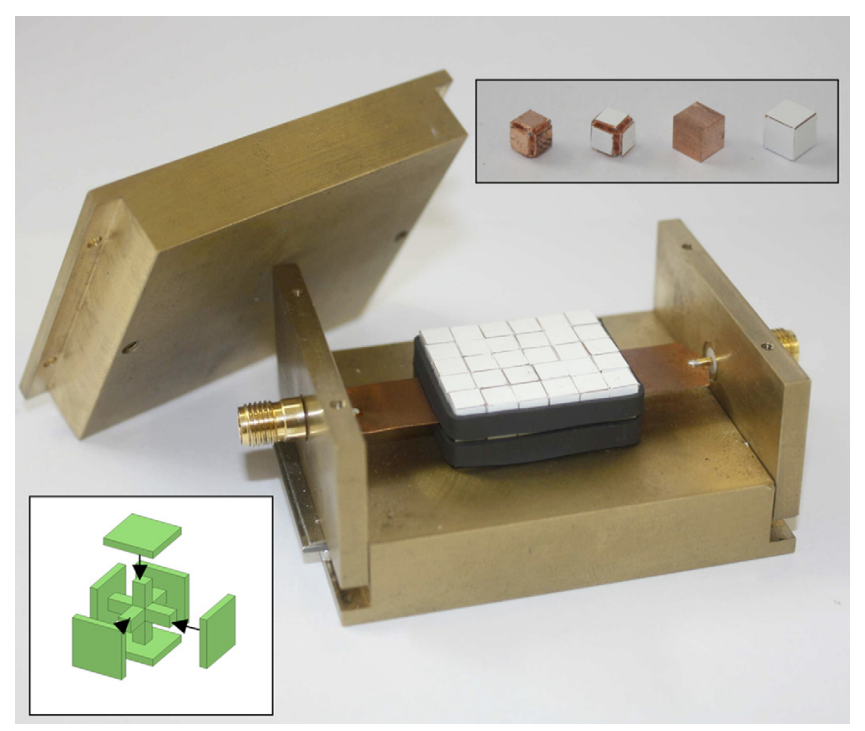

FIG. 1. A photograph of the stripline used to characterize the metamaterial samples, including the solid cube sample mounted in place. Inset top-right: individual solid and structured cubes with and without acrylic spacers. Inset bottom-left: exploded schematic of a single structured cubic element.
A stripline with a variable gap and easily interchangeable central conductor was fabricated such that the electromagnetic properties of a material of any height could be determined with only the fabrication of a new central conducting line of appropriate width being required to maintain the line at $50 \Omega$ (Figure 1). The impedance of this stripline was measured using an Anritsu Vectorstar VNA and found to vary between $44 \Omega$ and $55 \Omega$ over a frequency range of $10 \mathrm{MHz}$ to $4 \mathrm{GHz}$. After the electrical length of the stripline was determined by inspection of the phase of the transmission, the stripline was used as the "through" in a SOLT (short, open, load, through) calibration. The two arrays of cubes were subsequently placed in the gaps above and below the central conductor in the middle of the length of stripline oriented such that, along the wave propagation direction, the sample was 6 cubes long. The complex reflection and transmission coefficients were measured for radiation propagating through the sample in both directions and averaged in order to account for any misalignment of the sample. The phases of the amplitude coefficients obtained in this way are relative to the ends of the coaxial cables from the VNA, requiring the phase of the measured amplitude coefficients to be de-embedded to the face of the sample by multiplying the amplitude coefficients by $e^{2 k_{0} l}$ where $k_{0}$ is the free space wavevector and $l$ is the electrical distance from the input/output ports to the face of the sample $(l=($ electrical length $-d) / 2$, where $d$ is the length of the sample in the propagation direction).

The thin slab of material can be assumed to be a homogeneous material when the optical wavelength in the material is much greater than the size and spacing of the individual elements (in other words, we are truly in the metamaterial regime). Whilst one might expect the effective permittivity and permeability to be non-dispersive (since the materials of which they are fabricated are non-dispersive and the structure is far from resonance), when the electromagnetic wavelength within the metamaterial approaches the periodicity of the elements this will not be the case. Indeed, when the spacing is equal to a half of the electromagnetic wavelength in the metamaterial, a band gap will be evident, making it very clear that we have strayed from the metamaterial regime into that of diffractive optics. ${ }^{2}$ It is simple to determine the range of validity of the non-dispersive assumption by inspection of the reflected and transmitted intensities as a function of frequency. This is shown in Figure 2, along with the results of a finite element method (FEM) numerical model ${ }^{19}$ of the structure. The obvious discrepancy between the experimental and modeled data above approximately $4-5 \mathrm{GHz}$ is due to the stripline no longer being impedance matched at these higher frequencies. The slab of metamaterial forms a cavity that exhibits Fabry-Perot-like (standing wave) resonances due to interference between reflections from the front and back faces, which are manifested as reflection minima and transmission maxima. For a slab formed from a non-dispersive homogeneous medium, these resonant features would be equally spaced in frequency, and any deviation from this demonstrates that the material is dispersive. It can be seen from the inset of Figure 2 that the first and second order resonances are equally spaced and the material can thus be considered as homogeneous and non-dispersive up to approximately $5 \mathrm{GHz}$. This is beyond the range of 


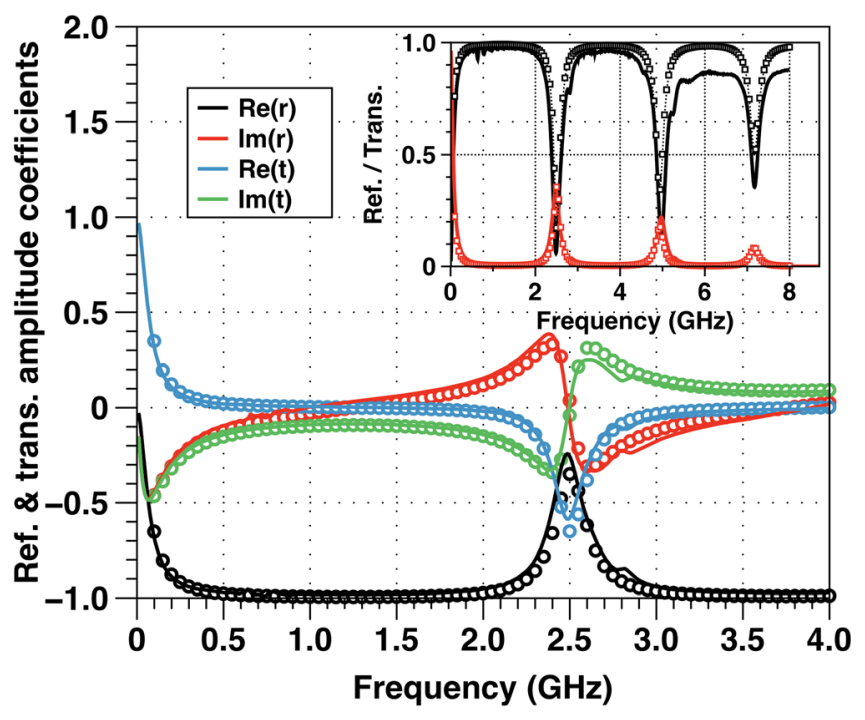

FIG. 2. Comparison between measured and fitted (using a Fresnel model) real and imaginary parts of the reflected and transmitted complex amplitude coefficients for the array of solid cubes (lines: experimental data and circles: model). Inset: Reflected and transmitted intensities as a function of frequency for the array of closely spaced solid metallic cubes (line). Also shown in the inset are modeled data obtained using a FEM numerical model (squares).

specification for the stripline $(10 \mathrm{MHz}$ to $4 \mathrm{GHz}$, dictated by geometrical dimensions and manufacturing tolerances).

As discussed previously, the NRW algorithm for determining the effective permittivity and effective permeability from the reflection and transmission complex amplitude coefficients is unsuitable for samples such as those studied here. Since we have already established that the permittivity and permeability are non-dispersive over the frequency range of interest, rather than trying to obtain the permittivity and permeability for each individual frequency (as in the NRW method), we are able to use the data for all frequencies simultaneously. This overcomes many of the limitations of the NRW method. Here, we have fitted the real and imaginary parts of the reflection and transmission amplitude coefficients to a three layer Fresnel model using the fmincon routine in MATLAB. ${ }^{20}$ The sum-of-squares difference between the Fresnel model and experimental data was used as the objective function with the only fitting variables being the real and imaginary parts of the permittivity and permeability (this method is similar to that employed by Abbas et al ${ }^{21}$ ). The resulting values were $\varepsilon=49.5+1.5 \mathrm{i}$ and $\mu=0.10+0 \mathrm{i}$, and the corresponding comparison of the experimental and modeled (Fresnel) complex amplitude coefficients are shown in Figure 2. The large reduction in the permeability due to the induced currents around the cubes is clearly evident and limits the refractive index to $\mathrm{n}=2.25+0.04 \mathrm{i}$.

We now turn our attention to an array of modified cubes. Following the suggestion of Shin et al., ${ }^{14}$ if each cube is replaced by an element consisting of six metal plates connected by 3 orthogonal metal wires intersecting at the center of the unit-cell (see inset Figure 1) the diamagnetic response can be significantly reduced whilst maintaining the strong capacitive response. Therefore, a metamaterial consisting of a regular array of such elements would be expected to exhibit a higher refractive index than the solid metal cube structure.
Such structured elements were fabricated using an additive-layer rapid-prototyping technique (Projet ${ }^{\mathrm{TM}} \mathrm{HD}$ 3000, 3D Systems Corporation, Rock Hill, USA), with each element subsequently coated with nano-crystalline copper (Morganic Metal Solutions, Farnham, UK), see inset Figure 1. Each structured cube had a side length of $4.2 \mathrm{~mm}$ (the same as the solid cubes), with each square metallic face being $2.8 \mathrm{~mm}$ long. The faces of each element had a square acrylic patch adhered to them so that when arranged into an ordered array they were uniformly separated. Two $1 \times 5 \times 6$ arrays of such elements were formed in the same way as for the solid cube sample and the same measurement and analysis method employed. The measured frequency dependent reflected and transmitted intensity, along with the corresponding FEM model, is shown in Figure 3. There are two important points to note when comparing this data to that for the solid cube sample. First, the low frequency edge of the band gap due to the periodicity is evident at approximately $4.8 \mathrm{GHz}$. It is clear, therefore, that the frequency range over which the permittivity and permeability can be considered as non-dispersive is significantly reduced compared to the solid cube metamaterial. By doubling the number of elements in the direction of propagation in the FEM modeling (doubling the thickness of the slab of material), the difference in frequency between successive resonances is reduced, demonstrating that the frequency range over which the non-dispersive assumption is valid is approximately $2 \mathrm{GHz}$ (inset Figure 3). Second, the difference between the measured and FEM modeled data becomes greater as the frequency approaches the lower edge of the band gap. This is due to a small difference in the spacing between elements in the propagation direction and in the direction parallel to the electric field. This anisotropy arises due to a slight compression of the sample within the stripline. This compression is required to ensure that any air gaps between the elements and the ground planes (which would significantly affect the effective permittivity of the structure) are minimised.

The comparison between the measured amplitude coefficients and the results from the Fresnel fitting is shown in

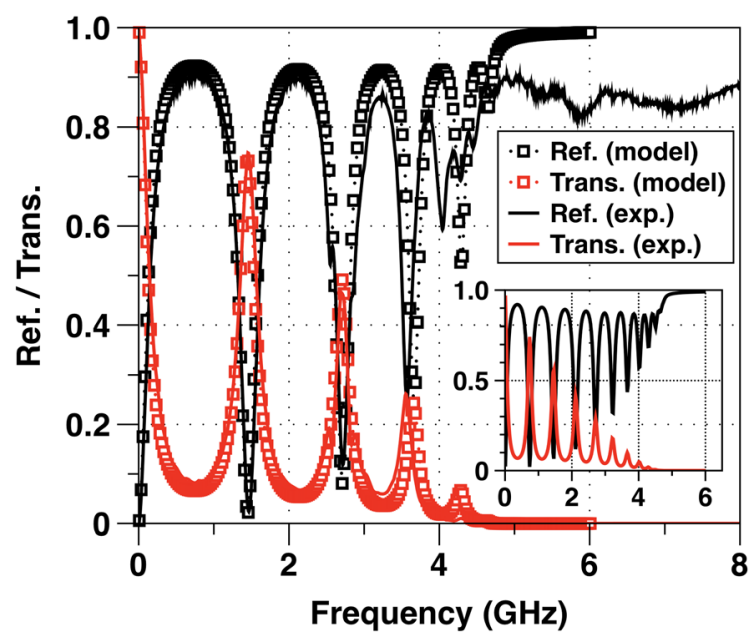

FIG. 3. Reflected and transmitted intensities as a function of frequency for the array of closely spaced structured metallic cubes. Also shown are modeled data obtained using a FEM model (see, Ref 19). Inset: FEM modeled data for a sample that is twice as thick in the direction of propagation. 


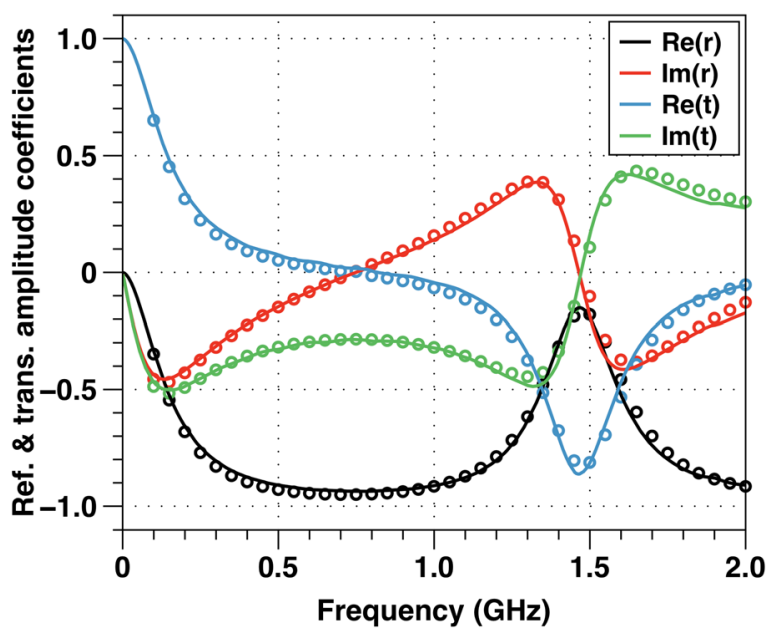

FIG. 4. Comparison between measured and fitted (using a Fresnel model) real and imaginary parts of the reflected and transmitted complex amplitude coefficients for the array of structured cubes (lines: experimental data and circles: model).

Figure 4 . The permittivity and permeability obtained for the structured cube sample were $\varepsilon=26.0+0.29 \mathrm{i}$ and $\mu=0.56$ $+0.01 \mathrm{i}$, corresponding to a refractive index of $\mathrm{n}=3.91$ +0.07 i. A comparison of the permittivity and permeability of the solid cubes with that of the structured cubes shows a reduction in the real part of the permittivity from nearly 50 to only 26 . Since the permittivity is predominantly due to the capacitative coupling between individual elements, it is highly sensitive to both the spacing between the elements and the size of the plates. Here, due to fabrication limitations, the plate size of the structured cubes is smaller than that of the solid cubes ( $2.8 \mathrm{~mm}$ compared to $4.2 \mathrm{~mm}$ ) and the permittivity is correspondingly reduced. Even though the permittivity of the structured cube sample is approximately half that of the solid cube sample, the refractive index is still nearly doubled due to the almost 6-fold increase in the permeability. This confirms the assertion by Shin et al. that the structuring of the cubic elements has reduced the diamagnetic response by limiting the area enclosed by the induced surface currents. In principle, the refractive index of a metamaterial formed from an array of appropriately structured cubes can be very high if the cubes can be arranged such that the gap between elements is very small, or through the inclusion of a higher refractive index material between the faces of the elements. FEM modeling has shown effective permittivities of several hundred are possible, corresponding to refractive indices of the order 20-30, but higher refractive indices could likely be achieved (these values are restricted by the modeling method, not the structures themselves). This work paves the way for further development of broadband metamaterials having structurally designed electromagnetic properties and extends the palette of materials available for devices requiring spatially engineered refractive index profiles such as those prescribed by the developing field of transformation optics.

This work was supported by the EPSRC through the QUEST Project (Ref: EP/I034548/1) and by BAE Systems through a company sponsored M.Phil.

${ }^{1}$ N. Engheta and R. W. Ziolkowski, Metamaterials: Physics and Engineering Explorations (Wiley, 2006).

${ }^{2}$ C. M. Soukoulis and M. Wegener, Science 330, 1633 (2010).

${ }^{3}$ D. R. Smith, J. B. Pendry, and M. C. K. Wiltshire, Science 305, 788 (2004).

${ }^{4}$ C. M. Soukoulis, J. Zhou, T. Koschny, M. Kafesaki, and E. N. Economou, J. Phys. Condens. Matter 20, 304217 (2008).

${ }^{5}$ D. R. Smith, W. J. Padilla, D. C. Vier, S. C. N. Nasser, and S. Schultz, Phys. Rev. Lett. 84, 4184 (2000).

${ }^{6}$ J. B. Pendry, Phys. Rev. Lett. 85, 3966 (2000).

${ }^{7}$ V. G. Veselago, Sov. Phys. Usp. 10, 509 (1968).

${ }^{8}$ J. B. Pendry, D. Schurig, and D. R. Smith, Science 312, 1780 (2006).

${ }^{9}$ U. Leonhardt, Science 312, 1777 (2006).

${ }^{10}$ U. Leonhardt and T. Tyc, Science 323, 110 (2009).

${ }^{11}$ D. F. Sievenpiper, E. Yablonovitch, J. N. Winn, S. Fan, P. R. Villeneuve, and J. D. Joannopoulos, Phys. Rev. Lett. 80, 2829 (1998).

${ }^{12}$ J. T. Shen, P. B. Catrysse, and S. Fan, Phys. Rev. Lett. 94, 197401 (2005).

${ }^{13}$ B. Wood and J. B. Pendry, J. Phys. Condens. Matter. 19, 076208 (2007).

${ }^{14}$ J. Shin, J. Shen, and S. Fan, Phys. Rev Lett. 102, 093903 (2009).

${ }^{15}$ M. Choi, S. H. Lee, Y. Kim, S. B. Kang, J. Shin, M. H. Kwak, K.-Y. Kang, Y.-H. Lee, N. Park, and B. Min, Nature 470, 369 (2011).

${ }^{16}$ W. Barry, IEEE Trans. Microwave Theory 34, 80 (1986).

${ }^{17}$ A. M. Nicolson and G. F. Ross, IEEE Trans. Instrum. Meas. 19, 377 (1970).

${ }^{18}$ W. W. Weir, Proc. IEEE 62, 33 (1974).

${ }^{19}$ ANSYS HFSs V14, Ansys Inc., Canonsburg, PA, 2011.

${ }^{20}$ MATLAB R2012a, The MathWorks Inc., Natick, MA, 2012.

${ }^{21}$ Z. Abbas, R. D. Pollard, and R. W. Kelsall, IEEE Trans. Instrum. Meas. 50, 1334 (2001). 